\title{
Synergism between upregulation of Rab7 and inhibition of autophagic degradation caused by mycoplasma facilitates intracellular mycoplasma infection
}

\author{
XIAOPENG HU ${ }^{1 *}$, JIE YU $^{2 *}$, XIANG ZHOU ${ }^{3},{\text { ZHAOMING } \mathrm{LI}^{4}, \mathrm{YUN} \mathrm{XIA}^{1}, \mathrm{ZHIYONG} \mathrm{LUO}^{1} \text { and YAQUN WU }}^{1}$ \\ ${ }^{1}$ Department of General Surgery, Tongji Hospital, Tongji Medical College, Huazhong University of Science and Technology, \\ Wuhan, Hubei 430030; ${ }^{2}$ First People's Hospital of Jiujiang City, Jiujiang, Jiangxi 330300; ${ }^{3}$ The Fifth Hospital of Huangshi City, \\ Huangshi, Hubei 435004; ${ }^{4}$ Department of Oncology, The First Affiliated Hospital, \\ Zhengzhou University, Zhengzhou, Henan 450052, P.R. China
}

Received May 2, 2013; Accepted January 10, 2014

DOI: $10.3892 / \mathrm{mmr} .2014 .1907$

\begin{abstract}
Following fusion of a mycoplasma with a host cell membrane, the inserted components of mycoplasma may then be transported through the endocytic pathway. However, the effects of mycoplasmas on the host cell endomembrane system are largely unknown. In this study, mycoplasma-induced changes in the dynamics of endocytic and autophagic systems were investigated. Endocytosis and autophagy are two major processes involved in the survival of intracellular prokaryotic pathogens. It was found that, immediately following infection, mycoplasmas induce endocytosis in the host cell; however, in the long term the mycoplasmas suppress turnover of the components of the endocytic pathway. Immunofluorescence microscopy revealed that Rab7 and LC3-II are recruited to the intracellular mycoplasma-containing compartments. Western blot analysis and quantitative reverse transcription-polymerase chain reaction (qPCR) showed that mycoplasmas increase expression of Rab7 by upregulating transcription, but increase levels of LC3-II and p62 by post-translational regulation. Furthermore, it was demonstrated that mycoplasma infection causes inhibition of autophagic degradation of LC3-II and p62. In addition, it was found that upregulation of Rab7 and inhibition of autophagic degradation synergistically contributes to intracellular mycoplasma accumulation. In conclusion, these findings suggest that mycoplasmas may manipulate host
\end{abstract}

Correspondence to: Dr Zhiyong Luo or Dr Xiaopeng $\mathrm{Hu}$, Department of General Surgery, Tongji Hospital, Tongji Medical College, Huazhong University of Science and Technology, 1095 Jiefang Avenue, Wuhan, Hubei 430030, P.R. China

E-mail: luozhiyong74@hotmail.com

E-mail: xiaopeng_hu@hust.edu.cn

*Contributed equally

Key words: mycoplasma, endocytic pathway, autophagy, Rab7, LC3, p62 cell endosomal and autophagic systems in order to facilitate intracellular infection.

\section{Introduction}

The lack of a cell wall renders mycoplasmas readily able to fuse with host cell membranes (1). The consequences of this remain largely unknown, since the fusion results in the insertion of various mycoplasmal cell components, complicating the interpretation of the pathogenesis (2-4). The fusion process has been observed using transmission electron microcopy (5). Following fusion with host plasma membrane or endosomal membrane, the mycoplasma membrane components integrate into the host cell membrane, along with membrane-associated proteins (1). These membrane-associated proteins are subsequently transported through the endocytic pathway.

The endocytic pathway is a highly dynamic membrane system consisting of a heterogeneous population of early, recycling and late endosomes. The endosomes receive the endocytosed membrane-bound vesicles and sort them for maturation, recycling or lysosomal degradation, respectively. It is currently proposed that the trafficking pathway of the endosomal system comprises three major processes: i) Constitutive recycling of early endosomes or intermediate endosomes to the plasma membrane; ii) fusion of multivesicular bodies (MVBs) with lysosomes for lysosomal degradation; and iii) fusion of MVBs with plasma membrane for exosomal release of intraluminal vesicles (6). All of these processes are crucial for cells to perform their normal physiological functions (7-9).

MVBs are morphologically distinct late endosomes that originate from an endosomal compartment through membrane invagination and the formation of intraluminal vesicles ranging between 50 and $100 \mathrm{~nm}$ in diameter (10). Prevention of the fusion between MVBs and lysosomes results in the inhibition of lysosomal degradation (9). The autophagic pathway, another evolutionarily conserved membrane system with pleiotropic functions, has a role in the cellular response to intracellular infection by which host cells eliminate invading microorganisms (11). MVBs and autophagosomes fuse prior to the lysosomal fusion step to form amphisomes (12). The 
endosomal system provides membrane components for autophagosome membrane biogenesis (13) and the amphisomes acquire machinery required for lysosomal fusion following the fusion of nascent autophagosomes with endosomes (14). The autophagosomal system overlaps extensively with the endosomal system and the autophagosome-lysosome fusion is a process that recapitulates endosome-lysosome fusion. Therefore, measuring the turnover of the substrates of autophagic degradation may partly reflect the rate of endolysosomal degradation.

Host cellular molecules, such as LC3, p62 and Rab7, are involved in the aforementioned processes of autophagic degradation. LC3-I undergoes post-translational modification to form lipidated LC3-II, an autophagosomal marker. p62 (SQTSM1), is an adaptor of LC3-II and serves as a substrate for autophagic degradation (15). Rab7 small GTPase contributes to the progression of the autophagy pathway and regulates trafficking of MVBs to lysosomes $(16,17)$. Numerous pathogenic microorganisms are capable of subverting host cellular endosomal and autophagosomal pathways in order to facilitate intracellular infection, often involving host cellular Rab family GTPases and LC3-II-positive vacuoles (18). For example, Mycoplasma pneumonia toxin induces the formation of vacuoles in host cells (19), which is associated with Rab GTPase functions. However, little is known about mycoplasma-induced modifications in the dynamics of the host cellular endomembrane system, or the host molecules involved in intracellular mycoplasma infection.

In this study, the effects of mycoplasma infection on the host cell endosomal and autophagosomal systems were investigated, as well as the molecular basis of intracellular mycoplasma accumulation involving Rab7 and autophagic markers LC3-II and p62.

\section{Materials and methods}

Antibodies and reagents. The primary antibodies used were as follows: LC3B (3868; Cell Signaling Technology, Inc. Beverly, MA, USA); p62/Sqstm1 (AT4033a; Abgent, San Diego, CA, USA); and Rab7 (55469-1-AP; Proteintech Group, Chicago, IL, USA). The secondary antibodies were obtained from Jiayuan Biotech (Wuhan, China). DiI was obtained from Molecular Probes (Carlsbad, CA, USA).

Cell culture. HeLa and SH-SY5Y cells were obtained from the American Type Culture Collection (Manassas, VA, USA) and maintained in Dulbecco's modified Eagle's medium (DMEM, high glucose; Hyclone, Logan, UT, USA) and a 1:1 ratio of DMEM/F12 medium (Gibco-BRL, Carlsbad, CA, USA), respectively. Culture medium was supplemented with $10 \%$ fetal bovine serum (FBS; Hyclone) and $2 \mathrm{mM}$ L-glutamate prior to use. The cells were cultured at $37^{\circ} \mathrm{C}$ in a humidified $5 \% \mathrm{CO}_{2}$ atmosphere.

Mycoplasma identification. The species of mycoplasma used were identified using polymerase chain reaction (PCR) with mycoplasma genus-specific degenerate primers, and followed by agarose gel electrophoresis, purification and T-vector cloning (pMD-18T vector, Takara, Dalian, China). The sequencing results confirmed that the mycoplasma contaminant was iden- tical to Mycoplasma pulmonis (M.pulmonis) UAB CTIP strain (NC_002771.1). The degenerate primers used for the conserved 16s-23s gene spacer region of the mycoplasma genus were as follows: forward 5'-ACACCATGGGAGYTGGTAAT-3', reverse 5'-CTTCWTCGACTTYCAGACCCAAGGCAT-3'.

Preparation of mycoplasma-containing cell culture supernatant. HeLa cells contaminated with Mycoplasma pulmonis were plated at a density of $1 \times 10^{5}$ cells $/ \mathrm{ml}$ in a six-well plate. The next day, the cells were washed three times with $0.01 \mathrm{M}$ phosphate-buffered saline (PBS) and incubated with $2 \mathrm{ml}$ DMEM without FBS for $24 \mathrm{~h}$. The cell culture supernatant was collected and cell debris was removed by filtration through a $0.8-\mu \mathrm{m}$ cut-off filter (Millipore, Bedford, MA, USA).

Quantitative PCR (qPCR) for mycoplasma and host cell genome copy numbers. For absolute qPCR, the pMD-18T plasmid (mycoplasmal sequence inserted) was diluted in a 1:10 serial dilution five times and utilized as the standard template The absolute copy number of mycoplasmas in the mycoplasma-containing cell culture supernatant was determined by comparison with the standard curve, and the multiplicity of infection (MOI) was determined accordingly.

For relative qPCR, the intracellular mycoplasma copy numbers were normalized to host genome copy numbers. The host cells were suspended in 0.01 M PBS and washed five times to remove extracellular planktonic mycoplasma. The genomic DNA was then extracted from total cell extracts and purified as previously described (20).

qPCR was performed using the LightCycler ${ }^{\circledR} 480$ Real-Time PCR Detection System (Roche, Basel, Switzerland) and an SYBR Green Real-Time PCR 2X premix kit (Takara). The reaction conditions were as follows: $15 \mathrm{sec}$ at $95^{\circ} \mathrm{C}$ followed by 45 cycles of denaturation for $20 \mathrm{sec}$ at $95^{\circ} \mathrm{C}$ and annealing and extension for $20 \mathrm{sec}$ at $60^{\circ} \mathrm{C}$. The melting curve of the products was determined and found to be specific. Primers used for the M. pulmonis 16s-23s gene spacer region were as follows: forward, 5'-GGAGCTGGTAATGCC CAAAGT-3' and reverse, 5'-ACGTTCTCGTAGGGATAC CTTG-3'. Primers for host cell genome ( $\beta$-hemoglobin) were as follows: forward, 5'-GAAGAGCCAAGGACAGGTAC-3' and reverse, 5'-CCAACTTCATCCACGTT CAC-3'.

$q P C R$. Total RNA was extracted from HeLa cells using TRIzol (Invitrogen, Carlsbad, CA, USA), and first strand cDNA reverse transcription was performed using a reverse-transcription RT-PCR kit (Takara) in the presence of Oligo (dT) 15 primers. qPCR was conducted using a LightCycler $\AA 480$ Real-Time PCR Detection System (Roche) and SYBR Green Real-Time PCR $2 \mathrm{X}$ premix kit (Takara). The reactions were performed under the following conditions: $15 \mathrm{sec}$ at $95^{\circ} \mathrm{C}$ followed by 40 cycles of denaturation for $15 \mathrm{sec}$ at $95^{\circ} \mathrm{C}$ and annealing and extension for $15 \mathrm{sec}$ at $60^{\circ} \mathrm{C}$. The melting curves for Rab7, LC3 and p62 were determined and found to be specific. A two standard curve method was used to quantify the expression levels of Rab7, LC3 and p62, and the mRNA levels were normalized to that of $\beta$-actin. Primers used for Rab7, LC3, p62 and $\beta$-actin were as follows: Rab7 forward 5'-CATCCTGGGAGATTCTGGAGTC-3' and reverse, 5'-TGT GTCCCATATCTGCATTGTG-3'; LC3 forward, 5'-GAGCAG 
CATCCAACCAAAATC-3' and reverse, 5'-GCCTGATTA GCATTGAGCTGTAAG-3'; p62 forward, 5'-GGACTTGGT TGCCTTTTCCAGTG-3' and reverse, 5'-GCAGCCGTC GCAGATCACAT-3'; $\beta$-actin forward, 5'-GCACCCAGC ACAATGAAGATC-3' and reverse, 5'-CTCGTCATACTC CTGCTTGCTG-3'.

Fluorescence microscopy. HeLa cells were plated on 0.17-mm coverslips or glass-bottomed Petri dishes for fluorescence microscopy. Images were captured using an Olympus FluoView $^{\mathrm{TM}}$ FV1000 confocal microscope (100X/1.40 oil lens) or an Olympus BX51 wide field upright microscope with a 60X 1.4 NA objective. DiI was used to label the cell membrane at $2 \mu \mathrm{M}$ for $15 \mathrm{~min}$ at $37^{\circ} \mathrm{C}$. HeLa cells expressing green fluorescent protein-tagged Rab7 (GFP-Rab7) were fixed by $4 \%$ paraformaldehyde (PFA) at room temperature for $10 \mathrm{~min}$, prior to the cells being observed by confocal microscopy. DAPI $(300 \mu \mathrm{M})$ was used to counterstain the nuclei for $10 \mathrm{~min}$ at room temperature. For immunostaining of Rab7 and LC3-II, HeLa cells were fixed by 4\% PFA and permeabilized by $0.1 \%$ Triton-X100 followed by blocking in 3\% bovine serum albumin (BSA). The cells were then incubated with Rab7 and LC3B primary antibodies at $4^{\circ} \mathrm{C}$ overnight in a humidified dark chamber. The following day, Alexa Fluor 488-labeled secondary antibodies were used to visualize the staining.

Western blot analysis. Western blot analyses were performed as previously described (21). For immunoblotting of LC3 in SH-SY5Y cells, mycoplasma-infected (infected at the MOI ratio of 10:1 for $24 \mathrm{~h}$ ) cells and control SH-SY5Y cells were serum starved for $2 \mathrm{~h}$ prior to treatment with $0.1 \%$ dimethylsulfoxide (DMSO) or Bafilomycin A1 (Baf A1; $10 \mathrm{nM}$ ) for $4 \mathrm{~h}$; and lysates were collected for western blot analysis against LC3. For western blot analysis of p62 levels, the cellular protein translation was blocked with $35 \mu \mathrm{M}$ cycloheximide (CHX) for 4,8 or $12 \mathrm{~h}$. The densitometry was analyzed using ImageJ software (Rasband WS, US National Institutes of Health, Bethesda, MD, USA). The densitometric ratio of LC3-II to $\beta$-actin band was expressed as fold-change relative to control cells treated with DMSO.

Plasmids, small interfering RNA (siRNA) and transfection. Human wild-type Rab7 coding sequence was cloned and inserted into EcoRI/BamHI site of pEGFP-C1 vector downstream of the GFP sequence in the right reading frame. siRNA duplexes were purchased from RiboBio Co., Ltd. (Guangzhou, China). HeLa cells were transfected with plasmids or siRNA duplexes (10 nM) using Lipofectamine ${ }^{\mathrm{TM}} 2000$ (Invitrogen) in accordance with the manufacturer's instructions. Rab7 and scrambled control siRNA duplexes were as described previously by Vanlandingham and Ceresa (16) with the modifications of two deoxythymidine added to the $3^{\prime}$ end of each sequence to make it more stable.

Statistical analysis. Data from three independent experiments are expressed as mean \pm standard deviation (SD). The Student's t-test was used for comparisons between different groups. In all cases, $\mathrm{P}<0.05$ was considered to indicate a statistically significant difference.

\section{Results}

Mycoplasmas affect the host cell endosomal system, promoting endocytosis and suppressing turnover of endocytic membranes. Mycoplasma membrane components are inserted into host cell membranes and endocytosed; as a result, mycoplasmas may interfere with host cell through the endocytic pathway. Therefore, changes elicited by mycoplasmas in the host cell endosomal system were investigated in this study.

In mycoplasma-infected HeLa cells, it was observed that the GFP-Rab7 vesicles accumulated in the peri-nuclear region $1 \mathrm{~h}$ post-infection with the mycoplasma, and that the GFP-Rab7 vesicles were increased in size compared with control cells (Fig. 1A). These morphological changes in late endosomal compartments indicate that the dynamics of the endosomal system were subverted by mycoplasma infection.

In order to investigate the mycoplasma-induced alterations in the dynamics of the endosomal system, chase assays for endocytosed membrane components in mycoplasma-infected HeLa cells and control cells were performed separately. Due to the fact that DiI is able to be embedded into lipid bilayers, and may be utilized as a marker for studying membrane orientation and fusion patterns, DiI was used to label the cell membrane for observation $(22,23)$. One hour after labeling, the endocytosed DiI-stained plasma membrane components were observed to have merged with the surrounding membrane of Rab7-positive compartments, which reflected the docking and/or fusing sites of DiI-stained endocytic vesicles with Rab7-positive late endosomes (Fig. 1A). However, after $72 \mathrm{~h}$, the DiI-stained vesicular structures were present in the lumen of Rab7-positive late endosomes. This phenomenon was more prominent in mycoplasma-infected cells compared with non-infected cells (Fig. 1A).

In mycoplasma-infected HeLa cells, the average fluorescence intensity of the DiI-stained endocytic vesicles within the peri-nuclear region (region of interest, ROI) per cell was four-fold higher than that in the control cells (Fig. 1B). In a prolonged observation, the membrane lipid dye that was transferred through the endocytic pathway persisted within the Rab7-positive compartments in the mycoplasma-infected HeLa cells with higher intensity relative to that in the control cells (Fig. 1B). The intracellular persistence of the DiI-bearing endosomal structures suggest that the elimination of endocytosed membrane components is suppressed in mycoplasma-infected HeLa cells.

In summary, the increase in the size of the endocytic vesicles indicates that mycoplasma may promote endocytosis immediately post-infection. The increased number and intensity of the DiI-bearing endocytic structures also indicates that mycoplasma infection suppresses turnover of the endocytosed membrane components in HeLa cells.

Mycoplasma infection upregulates Rab7 but appears to block the degradation of LC3-II and p62. Intracellular infection is associated with host cellular Rab7 and LC3, therefore, whether endogenous Rab7 and LC3-II were recruited to the surface of the intracellular mycoplasma-containing compartment was investigated. HeLa cells infected with M.pulmonis were immunostained for Rab7 and LC3-II. Confocal images revealed that, in mycoplasma-infected HeLa cells, the 


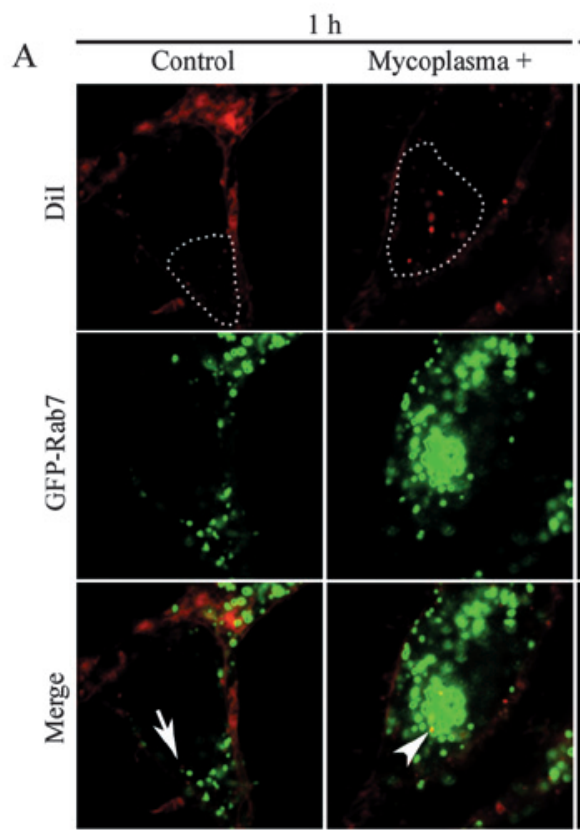

$72 \mathrm{~h}$

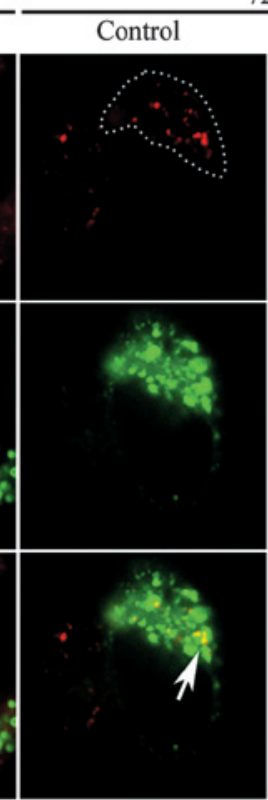

$\mathrm{h}$

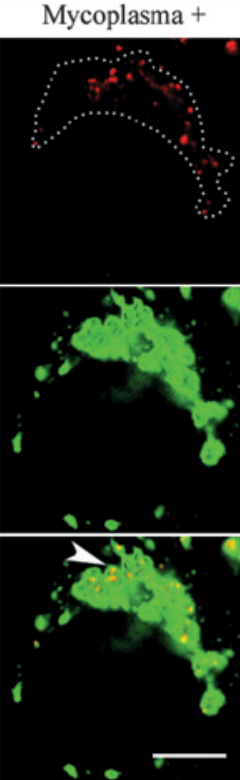

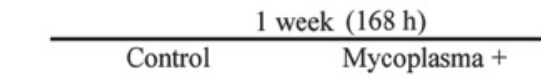

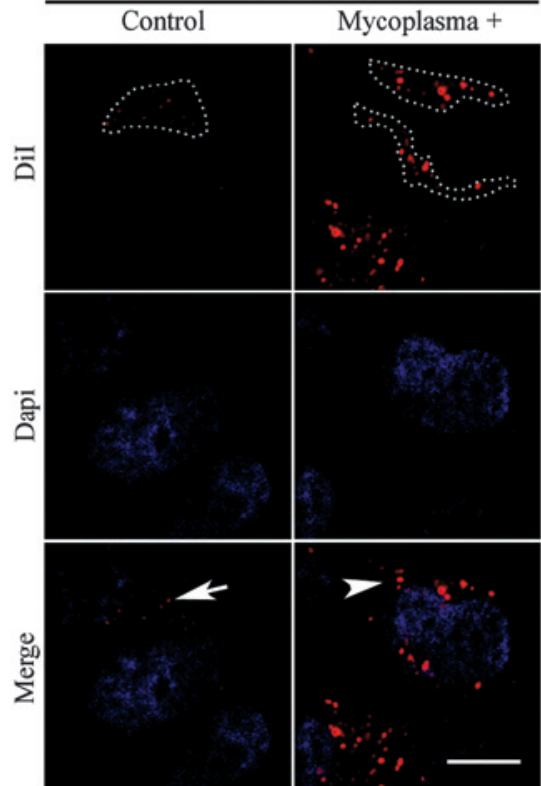

B

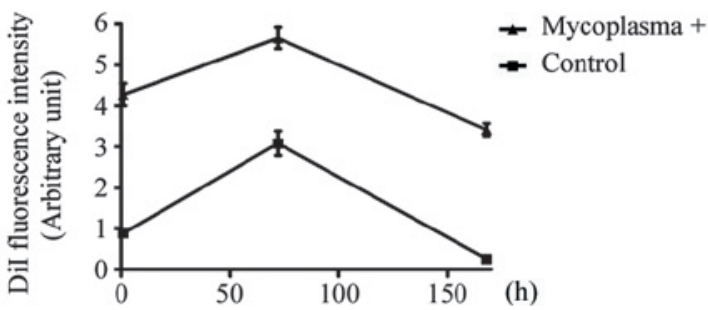

Figure 1. Mycoplasma infection affects the dynamics of the host cell endosomal system. (A) Cell culture supernatants from control and mycoplasma-positive HeLa cells were prepared as described in the Materials and methods. Mycoplasma-free HeLa cells transfected with green fluorescent protein-tagged Rab7 (GFP-Rab7) plasmids were labeled with membrane lipid dye DiI ( $2 \mu \mathrm{M})$ for 15 min followed by incubation with the control or the mycoplasma-positive cell culture supernatant for $45 \mathrm{~min}$. One hour following DiI labeling, the HeLa cells expressing GFP-Rab7 were observed by confocal microscopy. The cells treated in parallel were maintained in regular growth media until the observation at $72 \mathrm{~h}$ and one week after labeling. As the transient expression of GFP-Rab7 is not able to be observed in the long-term culture, DAPI staining was used to visualize the nucleus for observation at one week. The region of interest (ROI) for each cell is delineated by the white dotted line. The white arrows and arrow heads indicate the DiI-labeled vesicles in control and in mycoplasmacontaminated HeLa cells, respectively. The DiI-labeled vesicles were observed within the lumen of the Rab7-positive vacuoles at $72 \mathrm{~h}$ and persisted in the mycoplasma-contaminated HeLa cells for more than one week. The scale bar is $10 \mu \mathrm{m}$. (B) The DiI fluorescence intensity per cell within the ROI (observed at $1 \mathrm{~h}, 72 \mathrm{~h}$ and 1 week) was analyzed using ImageJ software (Rasband WS, US National Institutes of Health, Bethesda, MD, USA). The plotted data were from $\geq 30$ cells from three independent experiments. The error bar represents the mean \pm standard deviation.

extranuclear DAPI-positive particles were surrounded by membrane structures that were positively stained for Rab7 and LC3-II, and which were not observed in the negative controls (Fig. 2A and B). In addition, the fluorescence intensity of the endogenous Rab7 vesicles and LC3-II puncta/cell was higher in the mycoplasma-infected HeLa cells (data not shown). Therefore, Rab7 and LC3-II protein levels were investigated in HeLa cells contaminated with or without M.pulmonis. The levels of Rab7 and LC3-II were found to be upregulated in mycoplasma-infected HeLa cells compared with the negative control cells (Fig. 2C and D). The increased level of LC3-II in HeLa cells infected with $M$. pulmonis may result from either induction of autophagy or inhibition of lysosomal degradation; however, the accumulation of p62 (SQSTM1), which serves as a cargo adaptor for autophagic degradation $(24,25)$, indicated that it was the degradation process that was blocked in mycoplasma-infected HeLa cells (Fig. 2C and D). The expression levels of Rab7, LC3 and p62 mRNA were also measured using qPCR. The results showed that mRNA expression of Rab7 was upregulated following mycoplasma infection, whereas the mRNA expression of LC3 and p62 remained unchanged (Fig. 2E).

Mycoplasma infection results in inhibition of autophagic degradation. LC3-I is difficult to detect in HeLa cells by immunoblotting; therefore, SH-SY5Y cells, which express high levels of LC3-I, were used to investigate the alteration in LC3-II induced by $M$. pulmonis infection in the presence or absence of Baf A1. Prior to adding Baf A1, the cells were serum-starved to elicit an increase in autophagy. The results revealed that, in the mycoplasma-free cells, Baf A1 caused 2 .8-fold increase in the LC3-II level, whereas in the mycoplasma-infected cells only a slight increase in LC3-II level was observed (Fig. 3A), indicating that lysosomal degradation of LC3-II is blocked in mycoplasma-infected cells. Furthermore, in the presence of Baf A1, little difference was observed in LC3-II levels between mycoplasma-free and mycoplasma-infected cells (Fig. 3A), suggesting that induction of autophagy is not subverted by mycoplasma infection. These findings suggest that the mycoplasma-induced 


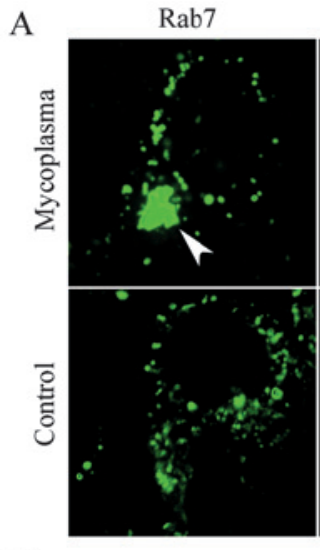

C

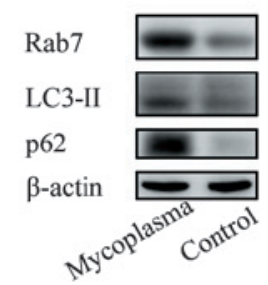

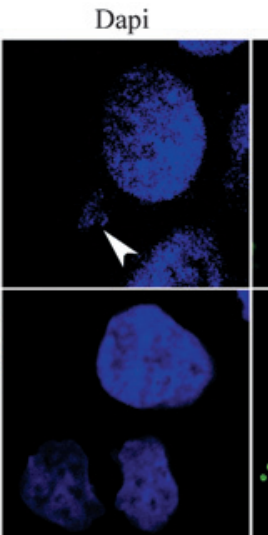

D

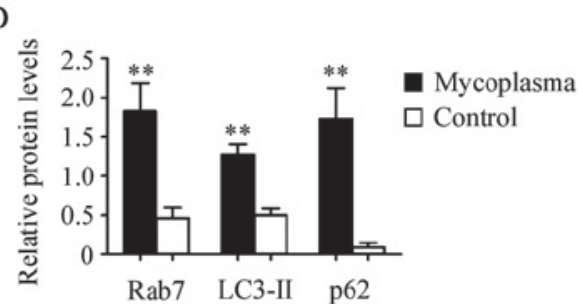

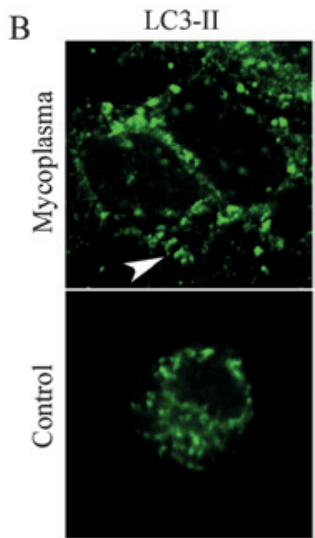
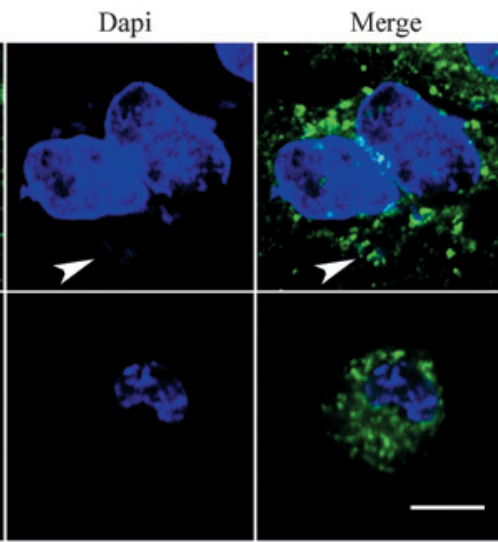

$\mathrm{E}$

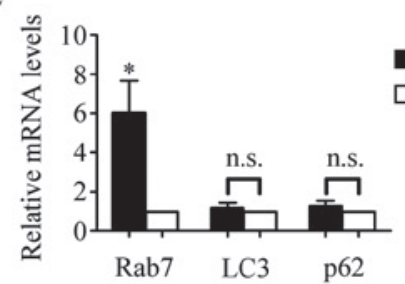

Figure 2. Effects of mycoplasma infection on the regulation of Rab7, LC3 and p62. HeLa cells contaminated with Mycoplasma pulmonis in long-term culture were immunostained for (A) Rab7 or (B) LC3-II and observed by confocal microscopy. The white arrow head indicates the extranuclear DAPI staining where endogenous Rab7 and LC3-II were recruited to the inclusion-like bodies. The scale bar is $10 \mu \mathrm{m}$. (C) HeLa cells contaminated with or without Mycoplasma pulmonis were harvested and subjected to western blot analysis for Rab7, LC3-II and p62. In addition, $\beta$-actin was used as a loading control. One representative result from three independent experiments is shown. (D) Relative protein levels of Rab7, LC3-II and p62, which are normalized to the densitometry of $\beta$-actin bands, from three independent experiments compared with control $\left({ }^{* *} \mathrm{P}<0.01\right)$. (E) Relative mRNA levels of Rab7, LC3 and p62, which are normalized to the mRNA levels of $\beta$-actin, from three independent experiments compared with control ( $(\mathrm{P}<0.05)$. Error bars represent the mean \pm standard deviation (SD). $\mathrm{n}$.s., not significant.

A
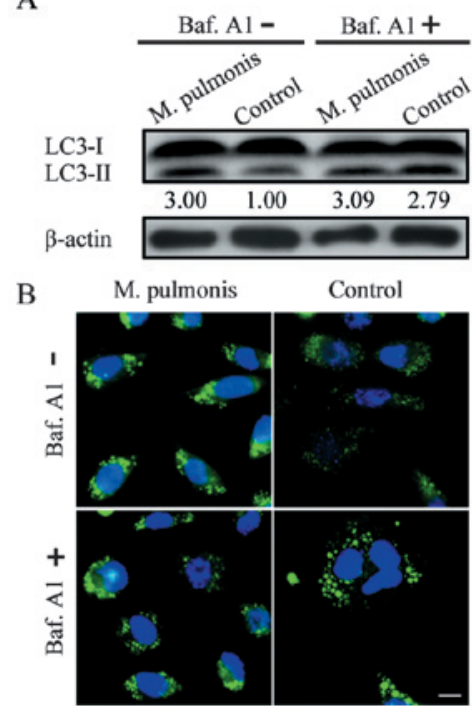

C
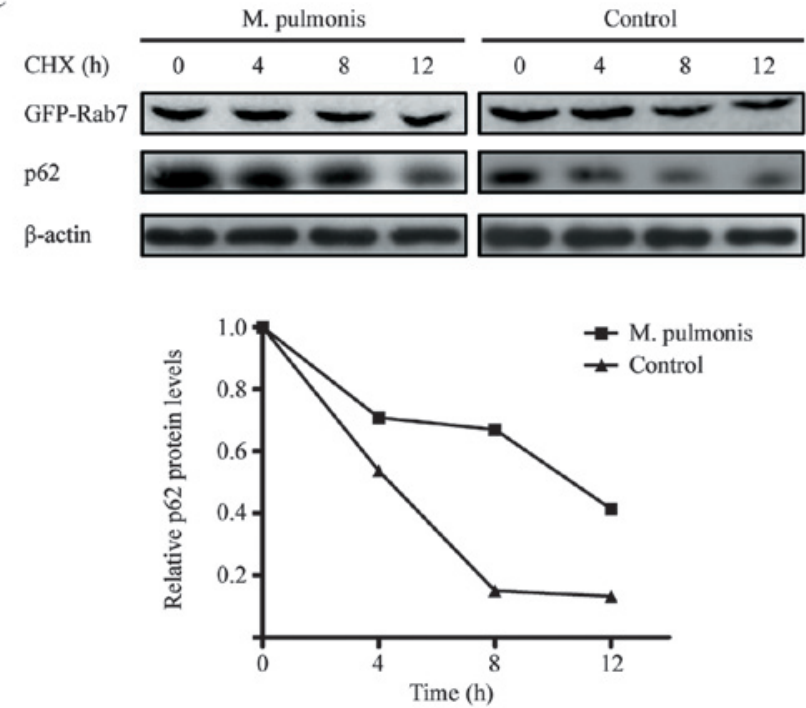

Figure 3. Mycoplasma infection suppresses autophagic degradation of LC3-II and p62. (A) SH-SY5Y cells were cultured with or without M. pulmonis. The cells were serum-starved for $2 \mathrm{~h}$ and then treated with or without $10 \mathrm{nM} \mathrm{Baf}$ A1 for $4 \mathrm{~h}$ prior to harvest. Western blot analysis against LC3 was performed on the cell lysates. One representative result from three independent experiments is shown. The numbers below the band represent the relative densitometric ratios of LC3-II/ $\beta$-actin, which was set to 1 in the control cell treated without Baf A1. (B) The cells treated in parallel to (A) were immunostained for LC3-II puncta and observed by a wide field fluorescent microscope (the scale bar represents $10 \mu \mathrm{m}$ ). (C) Mycoplasma-free HeLa cells were transfected with GFP-Rab7 plasmid, $6 \mathrm{~h}$ after the transfection the cells were infected with or without M. pulmonis (MOI 10:1) for an additional $24 \mathrm{~h}$. Then the cells were treated with or without $35 \mu \mathrm{M}$ CHX for 4,8 or $12 \mathrm{~h}$. The relative levels of $\mathrm{p} 62$ (densitometric ratio of $\mathrm{p} 62 / \beta$-actin) were identified by western blot analysis. The initial ratio at time zero was set to 1. One representative result from three independent experiments is shown. GFP, green fluorescent protein; MOI, multiplicity of infection; CHX, cycloheximide; Baf A1, Bafilomycin A1.

increase in LC3-II level was mainly due to the inhibition of autophagic degradation of LC3-II. In addition, observation of the LC3-II puncta by fluorescence microscopy revealed a similar pattern compared with the results of the western blot analysis (Fig. 3B). The degradation of p62 was also investigated using western blot analysis in a cycloheximide (CHX) 
A

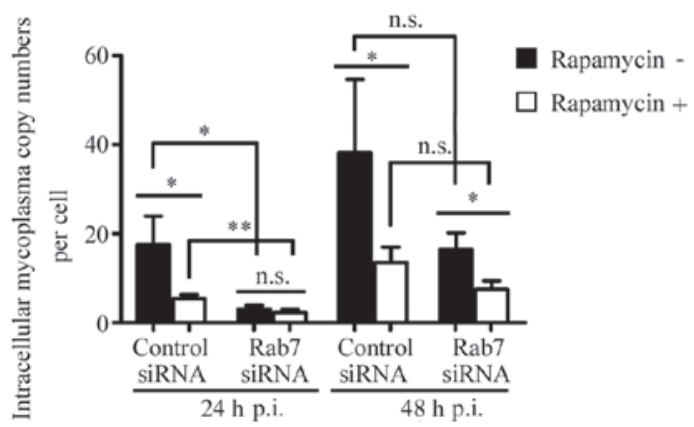

B

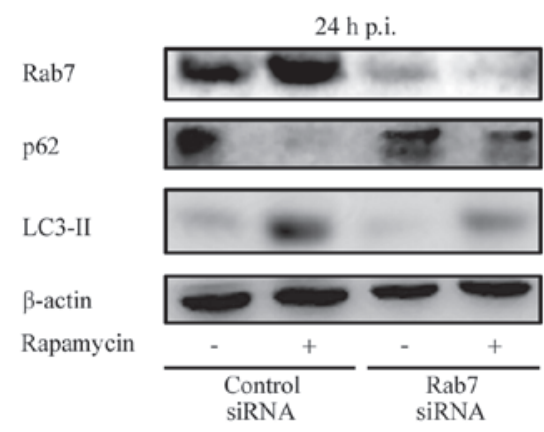

Figure 4. Upregulation of Rab7 combined with inhibition of autophagic degradation synergistically facilitate intracellular accumulation of mycoplasmas. (A) HeLa cells were transfected with Rab7 or control siRNA for $36 \mathrm{~h}$ followed by incubation with or without $100 \mathrm{nM}$ rapamycin for $12 \mathrm{~h}$. Rab7-depleted and control cells were then infected with cell culture supernatent containing M. pulmonis at an MOI of 10:1. The intracellular mycoplasma DNA copy numbers were determined using quantitative PCR at 24 and $48 \mathrm{~h}$ post-infection and normalized against the copy numbers of the host cell genome (n=3, t-test). "P<0.05, ${ }^{* *} \mathrm{P}<0.01$. (B) HeLa cells treated as in (A) were lysed at $24 \mathrm{~h}$ post-infection and subjected to western blot analysis with indicated antibodies. One representative result from three independent experiments is shown. siRNA, small interferring RNA; MOI, multiplicity of infection; PCR, polymerase chain reaction; p.i., post-infection. n.s., not significant.

chase assay in HeLa cells. Rab7 was found to be upregulated in mycoplasma-infected HeLa cells, which may have contributed to lysosomal degradation of p62 (17); therefore wild-type GFP-Rab7 was overexpressed in HeLa cells with or without mycoplasma infection to avoid bias. The results revealed that the degradation of p62 was inhibited in mycoplasma-infected HeLa cells, which was not rescued by overexpression of Rab7 (Fig. 3C).

Synergism between upregulation of Rab7 and inhibition of autophagic degradation is conducive to intracellular accumulation of mycoplasmas. Rab7 is involved in the intracellular infection of microorganisms (18); therefore, the effect of Rab7 on the intracellular accumulation of M. pulmonis was investigated. The results revealed that M. pulmonis DNA copies accumulated in HeLa cells at 24 and $48 \mathrm{~h}$ post-infection, and depletion of Rab7 by siRNA decreased the intracellular mycoplasma DNA copy numbers (Fig. 4A). Induction of autophagy by Rapamycin also attenuated the intracellular accumulation of M.pulmonis (Fig. 4A). Western blot analysis was utilized to confirm the efficiency of Rab7 knockdown in mycoplasma-infected HeLa cells $24 \mathrm{~h}$ post-infection (Fig. 4B).

\section{Discussion}

In this study, the effects of mycoplasma infection on the host cell endosomal system were investigated. In particular, mycoplasma-induced changes in the dynamics of endosomal membrane system, as well as in the autophagic degradation process, were studied. These are the two major processes involved in the survival of intracellular prokaryotic pathogens (26).

Following mycoplasma infection, Rab7-positive vacuoles were demonstrated to accumulate in the peri-nuclear region of host cells in the form of large spacious aggregates, which reflected the mycoplasma-induced alterations in the dynamics of the late endosomal system. The accumulation of large spacious late endosomes has also been observed in Salmonella infection, as well as in other microbial infections $(18,27)$.
It was hypothesized that mycoplasmas either promote the formation or inhibit the turnover of late endosomes, resulting in the formation of Rab7-positive large, spacious aggregates. Furthermore, semi-quantitative observation of DiI-bearing endocytic vesicles revealed that in mycoplasma-infected host cells the rate of internalization of plasma membrane is enhanced immediately following mycoplasma infection, and that the endocytosed vesicular structures are more resistant to mechanisms of elimination in a long-term culture. These findings suggest that mycoplasma infection inhibits the dynamic turnover of the endosomal system and increases endocytosis of the plasma membrane.

The net effect of mycoplasma-induced alterations in the dynamics of late endosomal turnover results in inhibition of downstream elimination of endocytic membrane components; however, whether the lysosomal degradation and exosomal discharge pathways are involved in the inhibition has yet to be elucidated.

The lysosomal degradation pathway, which involves the maturation of endosomes or autophagosomes followed by fusion with lysosomes, was studied and the role of the lysosomal pathway in mycoplasma-induced cellular responses was investigated. It was found that Rab7 and LC3-II were recruited to the mycoplasma-containing compartments, demonstrating an overlap between the endosomal and autophagosomal pathways during the cellular response to mycoplasma invasion. Western blot analysis, in combination with qPCR, revealed that Rab7 was transcriptionally upregulated, while LC3-II, as well as p62, were upregulated via post-translational mechanisms. Post-translational upregulation of LC3-II and p62 protein levels may result from inhibition of the autophagic flux $(15,24)$. It was therefore hypothesized that the lysosomal degradation process was suppressed by mycoplasma infection, resulting in the accumulation of the autophagosome marker LC3-II and the cargo protein p62, which explains the observed inhibition of endocytic membrane elimination.

The drug Baf A1 is used as a tool to block the autophagic degradation process (28). Using Baf A1 to block autophagic flux, it was found that inhibition in autophagic degradation 
was the main cause for mycoplasma-induced LC3-II accumulation. Following inhibition of protein synthesis by administration of $\mathrm{CHX}$, p62 proteins levels were chased. Rab7 contributes to maturation of late autophagic vacuoles (29), i.e., the fusion between autophagosomes and lysosomes, which facilitates the degradation of cargo proteins. However, the Rab7 protein expression levels are decreased in mycoplasma-free cells compared with mycoplasma-infected cells. Therefore, in this experiment, Rab7 was overexpressed to eliminate the influence of insufficient Rab7 levels on autophagic degradation of p62. Of note, it was found that mycoplasma infection suppressed cellular p62 degradation, despite the overexpression of Rab7, suggesting that fusion of autophagosomes with lysosome may be impeded by intracellular mycoplasma infection. This hypothesis may also apply to amphisome fusion with lysosomes, since it is also a late autophagic compartment resulting from autophagosome-endosome fusion (12).

In order to investigate the strategy used by the pathogen to manipulate the host cell for intracellular survival, the molecular basis underlying the intracellular accumulation of mycoplasmas was investigated. Rab7 was found to be required for the intracellular accumulation of mycoplasmas and it was revealed that the induction of autophagy contributes to reducing the intracellular mycoplasma accumulation. Upregulation of Rab7 may promote homotypic fusion between Rab7-positive compartments (16), which accounts for the formation of large spacious aggregates of Rab7-positive vacuoles. Therefore, it was hypothesized that the large spacious vacuoles, combined with the inhibition of autophagic degradation, may provide an niche for intracellular pathogens, thereby facilitating intracellular infection of mycoplasmas. Of note, DNA copy numbers were used to indicate that intracellular mycoplasma accumulation occurs regardless of the viability of mycoplasma. The observed increase in the intracellular accumulation of mycoplasmas may also be the result of increased endocytosis of mycoplasmas of extracellular origin rather than from intracellular replication; either explanationsappears consistent with our hypothesis.

In conclusion, mycoplasma infection interferes with the host endosomal system and perturbs the dynamics of the endosomes and autophagosomes by upregulating Rab7, as well as by suppressing autophagolysosomal degradation, resulting in an intracellular niche that may be exploited by mycoplasma or even other intracellular pathogens.

Phagolysosomal maturation arrest caused by intracellular infection of Mycobacterium tuberculosis may interfere with inward budding of human immunodeficiency virus (HIV) into multivesicular bodies (30). Similarly, the mycoplasma-induced alterations in the dynamics of the endosomal system may be involved in the pathogenic synergism of mycoplasma with other co-infecting pathogens. Moreover, Rab7, which may be upregulated by mycoplasma infection, is associated with entry of certain enveloped viruses into host cells $(31,32)$. Since other pathogens may also benefit from mycoplasma-induced alterations in the endosomal system, mycoplasmas may serve as helpers or cofactors for other co-infections of clinical significances via manipulation of the host cellular endosomal pathway, involving HIV and Mycobacterium tuberculosis.

\section{Acknowledgements}

This study was derived from a project funded by NSFC (National Natural Science Foundation of China).

\section{References}

1. Rottem S: Interaction of mycoplasmas with host cells. Physiol Rev 83: 417-432, 2003.

2. Paddenberg R, Weber A, Wulf S and Mannherz HG: Mycoplasma nucleases able to induce internucleosomal DNA degradation in cultured cells possess many characteristics of eukaryotic apoptotic nucleases. Cell Death Differ 5: 517-528, 1998.

3. Razin S, Yogev D and Naot Y: Molecular biology and pathogenicity of mycoplasmas. Microbiol Mol Biol Rev 62: 1094-1156, 1998.

4. Buim MR, Buzinhani M, Yamaguti M, et al: Mycoplasma synoviae cell invasion: elucidation of the Mycoplasma pathogenesis in chicken. Comp Immunol Microbiol Infect Dis 34: 41-47, 2011

5. Rottem S and Naot Y: Subversion and exploitation of host cells by mycoplasmas. Trends Microbiol 6: 436-440, 1998.

6. Simons $M$ and Raposo G: Exosomes - vesicular carriers for intercellular communication. Curr Opin Cell Biol 21: 575-581, 2009.

7. van Ijzendoorn SC: Recycling endosomes. J Cell Sci 119: 1679-1681, 2006

8. Keller S, Sanderson MP, Stoeck A and Altevogt P: Exosomes: from biogenesis and secretion to biological function. Immunol Lett 107: 102-108, 2006.

9. Luzio JP, Pryor PR and Bright NA: Lysosomes: fusion and function. Nat Rev Mol Cell Biol 8: 622-632, 2007.

10. Gruenberg J and Stenmark H: The biogenesis of multivesicular endosomes. Nat Rev Mol Cell Biol 5: 317-323, 2004.

11. Huang $\mathrm{J}$ and Brumell JH: Autophagy in immunity against intracellular bacteria. Curr Top Microbiol Immunol 335: 189-215, 2009.

12. Berg TO, Fengsrud M, Strømhaug PE, Berg T and Seglen PO: Isolation and characterization of rat liver amphisomes. Evidence for fusion of autophagosomes with both early and late endosomes. J Biol Chem 273: 21883-21892, 1998.

13. Longatti A, Lamb CA, Razi M, Yoshimura S, Barr FA and Tooze SA: TBC1D14 regulates autophagosome formation via Rab11- and ULK1-positive recycling endosomes. J Cell Biol 197: 659-675, 2012.

14. Mizushima N: Autophagy: process and function. Genes Dev 21: 2861-2873, 2007.

15. Klionsky DJ, Abeliovich H, Agostinis P, et al: Guidelines for the use and interpretation of assays for monitoring autophagy in higher eukaryotes. Autophagy 4: 151-175, 2008.

16. Vanlandingham PA and Ceresa BP: Rab7 regulates late endocytic trafficking downstream of multivesicular body biogenesis and cargo sequestration. J Biol Chem 284: 12110-12124, 2009.

17. Gutierrez MG, Munafó DB, Berón W and Colombo MI: Rab7 is required for the normal progression of the autophagic pathway in mammalian cells. J Cell Sci 117: 2687-2697, 2004.

18. Brumell JH and Scidmore MA: Manipulation of rab GTPase function by intracellular bacterial pathogens. Microbiol Mol Biol Rev 71: 636-652, 2007.

19. Johnson C, Kannan TR and Baseman JB: Cellular vacuoles induced by Mycoplasma pneumoniae CARDS toxin originate from Rab9-associated compartments. PLoS One 6: e22877, 2011.

20. Köck J, Rösler C, Zhang JJ, Blum HE, Nassal M and Thoma C: Generation of covalently closed circular DNA of hepatitis B viruses via intracellular recycling is regulated in a virus specific manner. PLoS Pathog 6: e1001082, 2010.

21. Luo X, Li Z, Li X, et al: hSav1 interacts with HAX1 and attenuates its anti-apoptotic effects in MCF-7 breast cancer cells. Int J Mol Med 28: 349-355, 2011.

22. Sund SE, Swanson JA and Axelrod D: Cell membrane orientation visualized by polarized total internal reflection fluorescence. Biophys J 77: 2266-2283, 1999.

23. Chen H, Kim S, Li L, Wang S, Park K and Cheng JX: Release of hydrophobic molecules from polymer micelles into cell membranes revealed by Forster resonance energy transfer imaging. Proc Natl Acad Sci USA 105: 6596-6601, 2008.

24. Mizushima N and Yoshimori T: How to interpret LC3 immunoblotting. Autophagy 3: 542-545, 2007. 
25. Bjørkøy G, Lamark T, Pankiv S, Øvervatn A, Brech A and Johansen T: Monitoring autophagic degradation of p62/SQSTM1. Methods Enzymol 452: 181-197, 2009.

26. Yamaguchi H, Nakagawa I, Yamamoto A, Amano A, Noda T and Yoshimori T: An initial step of GAS-containing autophagosome-like vacuoles formation requires Rab7. PLoS Pathog 5: e1000670, 2009.

27. Schroeder N, Mota LJ and Méresse S: Salmonella-induced tubular networks. Trends Microbiol 19: 268-277, 2011.

28. Yamamoto A, Tagawa Y, Yoshimori T, Moriyama Y, Masaki R and Tashiro Y: Bafilomycin A1 prevents maturation of autophagic vacuoles by inhibiting fusion between autophagosomes and lysosomes in rat hepatoma cell line, H-4-II-E cells. Cell Struct Funct 23: 33-42, 1998.
29. Jäger S, Bucci C, Tanida I, et al: Role for Rab7 in maturation of late autophagic vacuoles. J Cell Sci 117: 4837-4848, 2004.

30. Deretic V, Vergne I, Chua J, et al: Endosomal membrane traffic: convergence point targeted by Mycobacterium tuberculosis and HIV. Cell Microbiol 6: 999-1009, 2004.

31. Sieczkarski SB and Whittaker GR: Differential requirements of Rab5 and Rab7 for endocytosis of influenza and other enveloped viruses. Traffic 4: 333-343, 2003.

32. Vonderheit A and Helenius A: Rab7 associates with early endosomes to mediate sorting and transport of Semliki forest virus to late endosomes. PLoS Biol 3: e233, 2005. 